\title{
Tumor-suppressive function of methiothepin in human placental choriocarcinoma cells
}

\author{
Jin-Young Lee ${ }^{1}$, Whasun Lim(i) ${ }^{2}$ and Gwonhwa Song(ib ${ }^{3}$ \\ ${ }^{1}$ Department of Pharmacology and Toxicology, Medical College of Wisconsin, Milwaukee, Wisconsin, USA, \\ ${ }^{2}$ Department of Food and Nutrition, Kookmin University, Seoul, Republic of Korea and ${ }^{3}$ Department of \\ Biotechnology, Institute of Animal Molecular Biotechnology, Korea University, Seoul, Republic of Korea
}

Correspondence should be addressed to W Lim or G Song; Email: wlim@kookmin.ac.kr or ghsong@korea.ac.kr

\begin{abstract}
Placental choriocarcinoma is a malignant trophoblastic tumor associated with placentation. During placentation, complicated molecular networks are mediated by endocrine and paracrine signals. Serotonin neurotransmitters have been identified in the transmembrane region of human placental choriocarcinoma (HPC) cells as tumor promoters; therefore, their antagonists have anti-cancer properties. Although methiothepin, a serotonin receptor antagonist and FDA-approved psychotropic agent, has shown multi-pharmacological functions in various disease models, its anti-tumorigenic activity and mechanisms underlying its action against HPC are unknown. Therefore, we identified the anti-cancer effects of methiothepin in JEG3 and JAR HPC cells. Methiothepin attenuated mitochondrial function and induced endoplasmic reticular stress, reducing oxidative phosphorylation and causing metabolic shifting in HPC cells. Furthermore, methiothepin showed synergistic pharmacological effects with paclitaxel in HPC cells. Our results highlight the robust tumor-suppressive function of methiothepin in HPC. Our findings provide new insights into the repositioning of methiothepin from a psychotropic agent to novel anti-cancer agents, especially against HPC.

Reproduction (2020) 160 919-929
\end{abstract}

\section{Introduction}

In the early stages of embryo implantation, rapid cellular processes such as placentation, decidualization, and invasion occur (Carson et al. 2000), and this process involves a complicated interaction of molecular networks mainly mediated by endocrine and paracrine signals (Bowen et al. 2002). Because of the intricate interactions during placentation, even minor loss of proper control may lead to severe damages including either developmental failure or abortion, and chorionic trophoblast diseases such as placental choriocarcinoma (Al-Khan et al. 2013). Furthermore, oxidative stress induces defective placentation, and decreased oxidative phosphorylation (OXPHOS) is observed in placental dysfunction (Yung et al. 2019). Considering the high demand for ATP synthesis during placentation, this may be linked to excessive mitochondrial respiration; hence, regulation of mitochondrial bioenergetics may contribute to the treatment of placental choriocarcinoma (Rosario et al. 2019). Therefore, we focused on OXPHOS- and mitochondrial electron transport chain (ETC) activity-associated anti-cancer mechanisms of the serotonin receptor antagonist methiothepin, which is an FDA (Food and Drug Administration)-approved psychotropic agent.
Serotonin (5-HT, 5-hydroxytryptamine) is primarily known as a neurotransmitter hormone, but several studies have identified its function as a growth simulator with mitogenic effects in various type of reproductive cells, including rat jejunal crypt cells (Tutton \& Barkla 1987), bovine placental cells (Fecteau \& Eiler 2001), and fetal tissues (Lauder et al. 1981). Besides, it has also been described as a tumor promoter in diverse types of tumorigenesis, such as in adenocarcinoma (Tutton \& Barkla 1987), pancreatic carcinoid cells (Ishizuka et al. 1992), and small cell lung cancer (Cattaneo et al. 1993). In human trophoblast and choriocarcinoma cell lines, 5-HT receptor has been characterized at the plasma membrane, and cell proliferation was stimulated by 5-HT through mitogenic function (Sonier et al. 2005). 5-HT stimulation also shows high energy demand, which leads to oxygen consumption rate (OCR) upregulation and increased ATP production, thus enhancing mitochondrial function in cortical neurons (Fanibunda et al. 2019). In agreement with this overall concept, 5-HT receptor antagonists have been described as antitumorigenic factors and apoptosis inducers, whereas selective agonists of 5 - $\mathrm{HT}$ promote tumor growth through mitogenic factors (Ataee et al. 2010). Methiothepin is an FDA-approved psychotropic agent, as well as a 5-HT receptor antagonist known to regulate gastrointestinal 
function (Pithadia \& Jain 2009). However, none of the previous studies have elucidated the anti-carcinogenic role of 5-HT antagonists in placental choriocarcinoma.

Therefore, we investigated the tumor-suppressive function of the 5-HT antagonist methiothepin in JEG3 and JAR placental choriocarcinoma cells. With our novel findings, we aim to: (1) identify the anti-tumorigenic effects of methiothepin by inducing proliferation and apoptosis in JEG3 and JAR human placental choriocarcinoma cells; (2) demonstrate mitochondrial dysfunction and calcium disruption by methiothepin; (3) elucidate the effect of methiothepin on metabolic processes including mitochondrial respiratory chain activity; (4) evaluate the endoplasmic reticulum (ER) activity induced by methiothepin and (5) investigate methiothepin-associated sensitization to paclitaxel in JEG3 and JAR choriocarcinoma cells.

\section{Materials and methods \\ Chemicals and antibodies}

Methiothepin, Hoechst 33342 stain, and propidium iodide were purchased from Sigma-Aldrich, and Z-VAD-FMK was purchased from Calbiochem. Antibodies were acquired from Cell Signaling Technology.

\section{Cell maintenance and chemical treatment}

The human placental choriocarcinoma cell lines JEG3 and JAR were purchased from the American Type Culture Collection (ATCC). Cells were maintained in RPMI 1640 medium containing 10\% fetal bovine serum (FBS; HyClone Laboratories, South Logan, UT, USA) and $1 \%$ penicillin-streptomycin and incubated at $37^{\circ} \mathrm{C}$ under $5 \% \mathrm{CO}_{2}$ conditions. For chemical treatment, cells were seeded at a density of 20,000 cells $/ \mathrm{cm}^{2}$ on the day prior to the treatment, and treated according to the indicated conditions.

\section{Analysis of cell viability and proliferation}

Cell viability and proliferation were measured by trypan blue exclusion assay, as described previously (Lee et al. 2020a). In detail, cell viability was calculated as the ratio of living cells to total cells, the sum of living and dead cells, and cell proliferation was calculated as the proportion of living cells. In addition, CellTiter-Glo Luminescent assay (Promega) was used to measure intracellular ATP production, and the specific method was followed according to the manufacturer's recommendations.

\section{Analysis of caspase activation and apoptosis by methiothepin}

Hoechst 33342 (Cat. No: B2261) and propidium iodide (Cat. No: P4170) were used to verify DNA fragmentation in apoptotic cells. FITC Annexin V Apoptosis Detection Kit (BD Bioscience, Franklin Lakes, NJ, USA) was used to detect specific cell death modalities. Protocols were followed according to the manufacturer's instructions. Caspase-Glo 3/7 assay (Promega) and Z-VAD FMK (Calbiochem) to detect caspase activities. Specific protocols were followed as described previously (Lee et al. 2020a).

\section{Measurement of mitochondrial dysfunction and calcium dysregulation in cytosol}

JC-1 and Fluo-4 fluorescence dyes (Sigma-Aldrich) were used to detect mitochondrial membrane potential and mitochondrial calcium levels, respectively. Cells were treated with methiothepin for $24 \mathrm{~h}$ at indicated concentrations and then stained with the fluorescence dyes. After incubation, cells were washed several times and detected using FACS. The protocols were followed as described previously (Lee et al. 2020a).

\section{Western blot analysis}

Proteins from JEG3 and JAR cells were extracted from the whole-cell lysates of methiothepin-treated cells. Protein concentration was determined by the Bradford protein assay (Bio-Rad). For western blotting analysis, a specific protocol described previously was followed (Lee et al. 2020a). Chemiluminescent signals of the blots were detected using SuperSignal West Pico, and visualized using the ChemiDoc EQ system and Quantity One software (Bio-Rad).

\section{Mitochondrial stress analysis}

To analyze the mitochondrial stress, we evaluated the OCR and used a series of inhibitors including oligomycin, FCCP, rotenone, and antimycin (mitochondrial complex I and III inhibitors) to assess OXPHOS levels. Seahorse mitochondrial stress analysis kit was purchased from Agilent Technologies, and experiments were performed according to the manufacturer's recommendations.

\section{Statistical analysis}

Statistical analysis was calculated by one-way or two-way ANOVA. Post hoc analyses were calculated by using GraphPad Prism 7 software. Statistical significances were indicated by $P$-values $<0.05$ and are presented as follows in the legend: ${ }^{*} P<0.05,{ }^{* *} P<0.01,{ }^{* * *} P<0.001$; Dunnet's post hoc analysis and Sidak's post hoc analysis). All histograms are presented as the means \pm S.D. of three independent experiments.

\section{Results}

\section{Methiothepin mitigates the proliferation of JEG3 and JAR human placental choriocarcinoma cells and induces DNA fragmentation and caspase activation}

For the primary optimization of the experimental methiothepin concentration, we determined the cell viability of JEG3 and JAR cells treated with various concentrations of methiothepin for $24 \mathrm{~h}$. $\mathrm{LC}_{50}$ 
concentrations at $24 \mathrm{~h}$ were determined at 11.4 and $8.6 \mu \mathrm{M}$ in JEG3 and JAR cells, respectively (Fig. 1A and B). Based on this information, we evaluated the effects of methiothepin on cell proliferation at concentrations of 0 to $15 \mu \mathrm{M}$ for 24 to $72 \mathrm{~h}$. The results showed that methiothepin dose-dependently suppressed JEG3 and JAR cell growth (Fig. 1A and B). In addition, cell viability was diminished by 53.4 and $42.7 \%$ in JEG3 (Fig. 1C) and JAR (Fig. 1D) cells, and intracellular ATP levels were more markedly decreased by 34.1 and $18.2 \%$ in both JEG3 (Fig. 1C) and JAR (Fig. 1D) cells by the highest concentration methiothepin. These results suggest that methiothepin primarily regulates ATP production in choriocarcinoma cells, thereby leading to the suppression of cell proliferation. We further confirmed the effects of methiothepin on DNA fragmentation in both placental choriocarcinoma cells by using Hoechst and PI double staining analysis. In JEG3 cells treated with $15 \mu \mathrm{M}$ methiothepin, chromatin condensation and PI-positive cells were observed, but not in the vehicle treatment (Fig. 1E). For JAR cells, DNA fragmentation was mainly caused by methiothepin, and no PI-positive cells were detected (Fig. 1F). The above results suggest that methiothepin inhibits the proliferation of JEG3 and JAR cells.

\section{Methiothepin promotes mitochondrial apoptosis in human placental choriocarcinoma cells through the regulation of apoptosis-associated proteins}

As our results from Hoechst and PI staining implied that methiothepin-induced cell death mechanisms may be slightly different between JEG3 and JAR cells, we conducted Annexin $\mathrm{V}$ and $\mathrm{PI}$ analysis to confirm this. According to our data, methiothepin increased the proportion of cells showing early apoptosis, consequently leading to an increase in the proportion of JEG3 cells showing late-apoptosis (Fig. 2A). In particular, $15 \mu \mathrm{M}$ methiothepin induced early- and late-apoptosis by 19 and 41\%, respectively. Similarly, methiothepin also stimulated early- and late-apoptosis by 9 and 30\%, respectively, in JAR cells (Fig. 2B). In both types of placental choriocarcinoma cells, methiothepin increased the extent of apoptosis, but showed different cell death modalities in JEG3 and JAR cells. We then used the caspase 3/7 luminescence assay to analyze whether
A

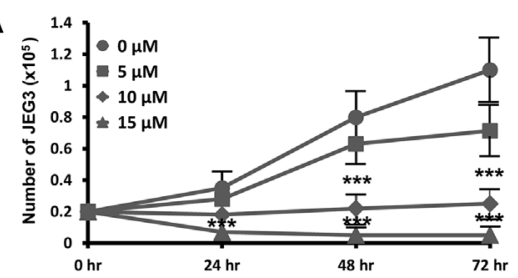

C

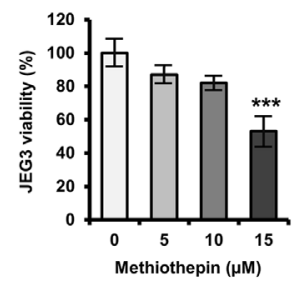

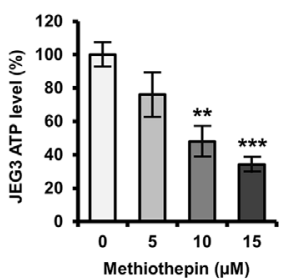
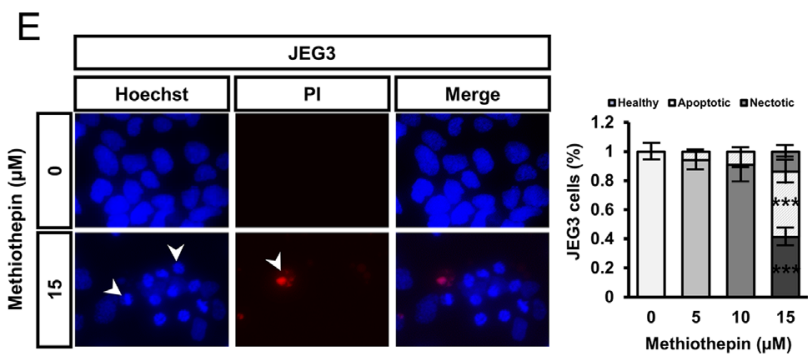

$\mathrm{B}$

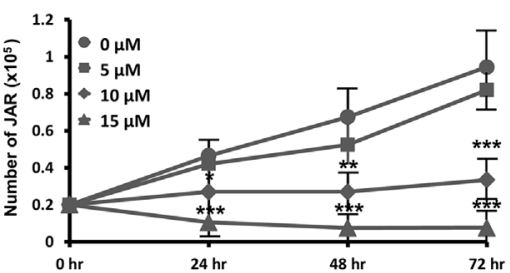

D
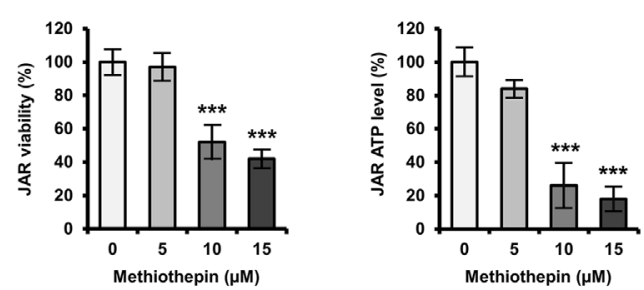

$\mathrm{F}$

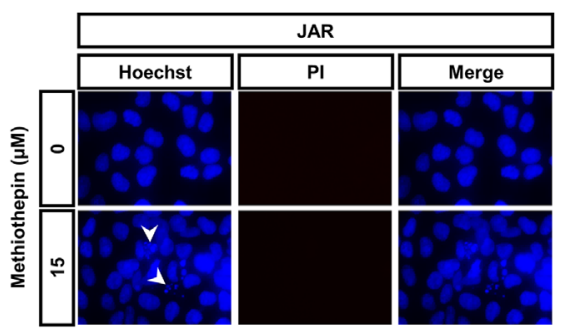

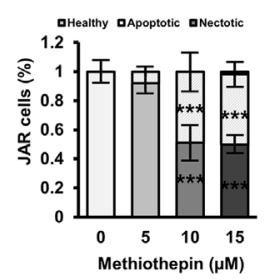

Figure 1 Anti-cancer effects of methiothepin in human placental choriocarcinoma cells. (A and B) Proliferation was determined by trypan blue exclusion analysis on JEG3 (A) and (B) JAR cells treated with methiothepin at indicated concentrations. (C and D) MTT assay (left panel) and Cell Titer-Glo Luminescent assay (right panel) confirming the effect of various concentrations of methiothepin on JEG3 (C) and JAR (D) viability (left panel) and intracellular ATP levels (right panel), respectively. (E and F) Hoechst and PI double staining conducted to identify DNA fragmentation with and without methiothepin in JEG3 (E) and JAR (F) cells. Fluorescent images were obtained by microscopy (left panel) and quantification was determined by the average number of cells by cell counting (right panel) in JEG3 (E) and JAR (F) cells. Results are presented as means \pm S.D. obtained from three independent experiments. The asterisk indicates statistically significant differences analyzed by Dunnett's test, one-, or two-way ANOVA $\left({ }^{*} P<0.05 ;{ }^{* *} P<0.01 ;{ }^{* * *} P<0.001\right)$. 
A

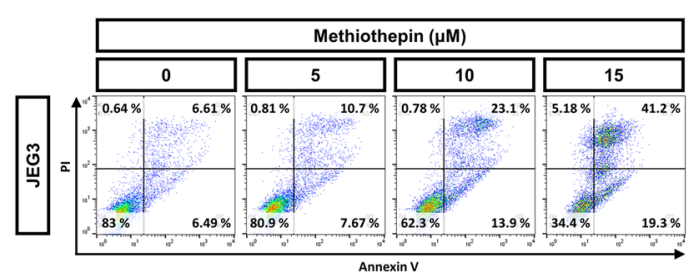

B

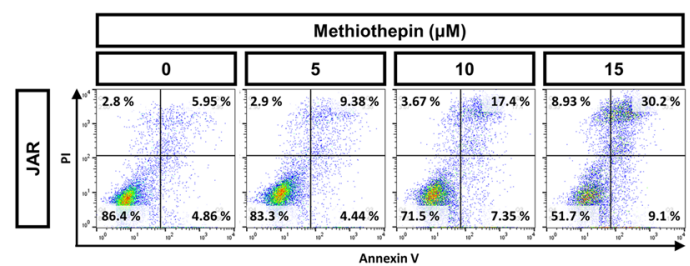

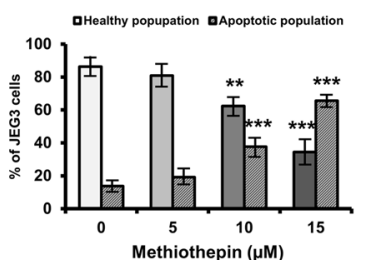

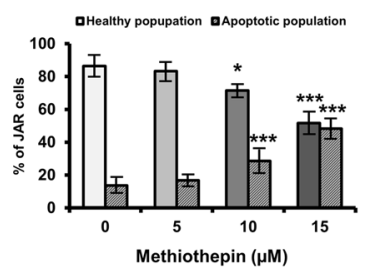

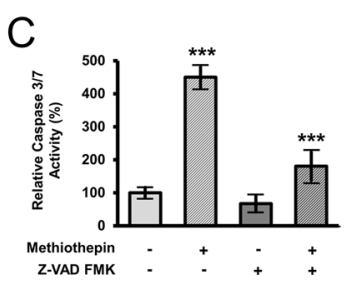

D

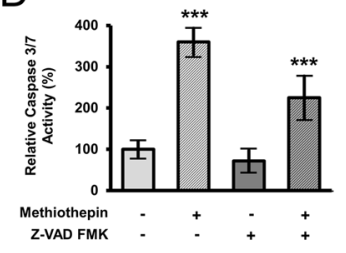

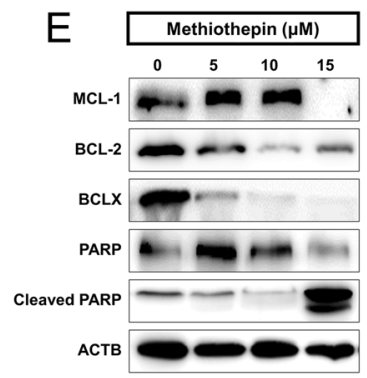
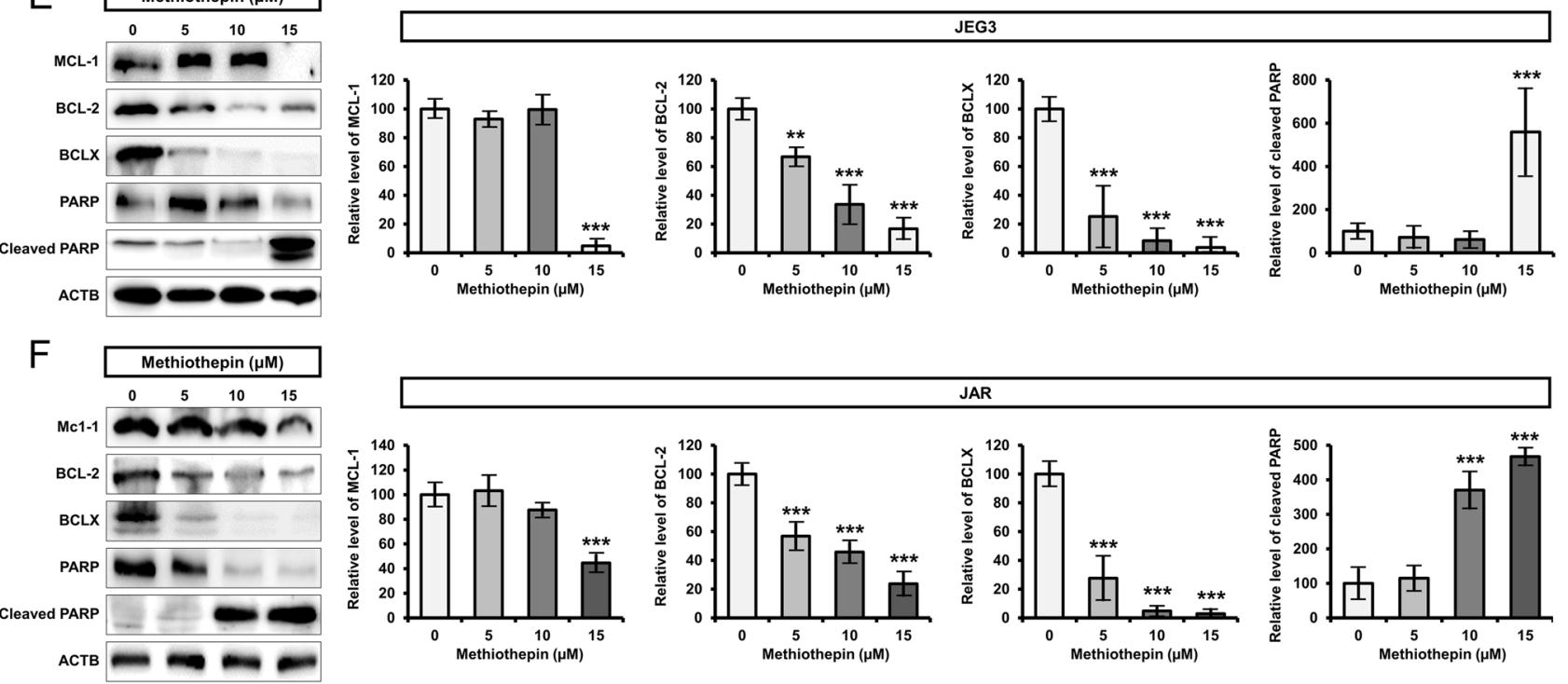

Figure 2 Effect of methiothepin on apoptosis and apoptosis-associated protein expression in human placental choriocarcinoma cells. (A and B) Methiothepin-induced cell death modality determined by Annexin $\mathrm{V}$ and PI staining and detected by flow cytometry (left panel) and quantification (right panel) in JEG3 (A) and JAR (B) cells. (C and D) Caspase 3 and 7 activities evaluated at indicated conditions of JEG3 (C) and JAR (D) cells using Caspase-Glo 3/7 assay. Z-VAD- FMK was applied as a pan-caspase inhibitor to evaluate the caspase dependencies. (E and F) Immunoblot (left panel) and densitometry analysis (right panel) of JEG3 (E) and JAR (F) cells by dose-dependent methionine treatment. Results are presented as means \pm S.D.. obtained from three independent experiments. Proteins of MCL-1, BCL-2, BCLX were validated as anti-apoptotic markers, and PARP cleavage was tested as a pro-apoptotic marker. The asterisk indicates statistically significant differences analyzed by

Dunnett's test, one -way ANOVA $\left({ }^{*} P<0.05 ;{ }^{* *} P<0.01 ; * * P<0.001\right)$.

methiothepin-triggered DNA fragmentation depends on caspase activation. Methiothepin in JEG3 and JAR cells increased the activity of caspase by 4.4 and 3.6 times, respectively; nevertheless, its activation was overturned by the pan-caspase inhibitor, Z-VAD-FMK (Fig. 2C and D). Combining the results of Annexin PI staining in Fig. 2A and $\mathrm{B}$ and the results of caspase activity in Z-VAD in Fig. $2 C$ and $D$, it is speculated that methiothepin significantly induces apoptosis in a caspase-dependent manner in human placenta choriocarcinoma cells. To elucidate the mechanisms of methiothepin-induced apoptosis, we evaluated the expression of proteins mainly associated with mitochondria-dependent apoptosis regulation. For this experiment, the levels of both anti-apoptotic proteins (MCL-1, BCL-2, and BCLX) and pro-apoptotic proteins (PARP) were analyzed in response to varied doses of methiothepin. In JEG3 cells, MCL-1, BCL-2, and BCLX expression were shown to decrease by 21.1-, 16.7-, and 3.6-fold, respectively, at the highest concentration of methiothepin, compared to vehicle treatment (Fig. 2E). In contrast, PARP cleavage increased by 5.6-fold with 15 $\mu \mathrm{M}$ methiothepin compared to the control. In JAR cells, the protein expression of MCL-1, BCL-2, and BCLX was suppressed by 2.3-, 4.2-, and 34.4-fold, respectively, whereas PARP cleavage was stimulated by 4.7 -fold following methiothepin treatment (Fig. 2F). 


\section{Methiothepin increases mitochondrial dysfunction and mitochondrial calcium overload}

We have confirmed that methiothepin induces caspasedependent apoptosis in placental choriocarcinoma cells, and this particular cell death mechanism is known to be regulated primarily by mitochondria (Matsuyama \& Reed 2000). We then investigated the effects of methiothepin on mitochondria, assuming that it would be caused by mitochondrial stress. In order to explore our hypothesis, we first determined the effects of methiothepin on mitochondrial membrane potential in both choriocarcinoma cells. Mitochondrial membrane potential $(\Delta \psi \mathrm{m})$ was maximally increased by 1.6 -fold in JEG3 cells and 2.6-fold in JAR cells by methiothepin (Fig. 3A and B). These results indicated that significant depolarization of mitochondrial membrane occurred by treatment with methiothepin in both placental choriocarcinoma cell types. In addition, we analyzed the effects of methiothepin on mitochondrial calcium influx by using Fluo-4 AM, a fluorescence calcium indicator. Interestingly, calcium influx into the mitochondria was maximally increased by 1.8 -fold in JEG3 cells, but not in JAR cells (Fig. 3C and D). In JEG3 cells, once choriocarcinoma cells are exposed to methiothepin, calcium influx from the cytosol to mitochondria occurs along with increased depolarization of the inner mitochondrial membrane, leading to mitochondrial rupture, proton leakage, and caspase activation, subsequently stimulating the apoptosis pathway. However, In JAR cells, mitochondrial membrane depolarization was observed and more sensitively ruptured rather than JEG3 cells by methiothepin but did not lead to calcium efflux.
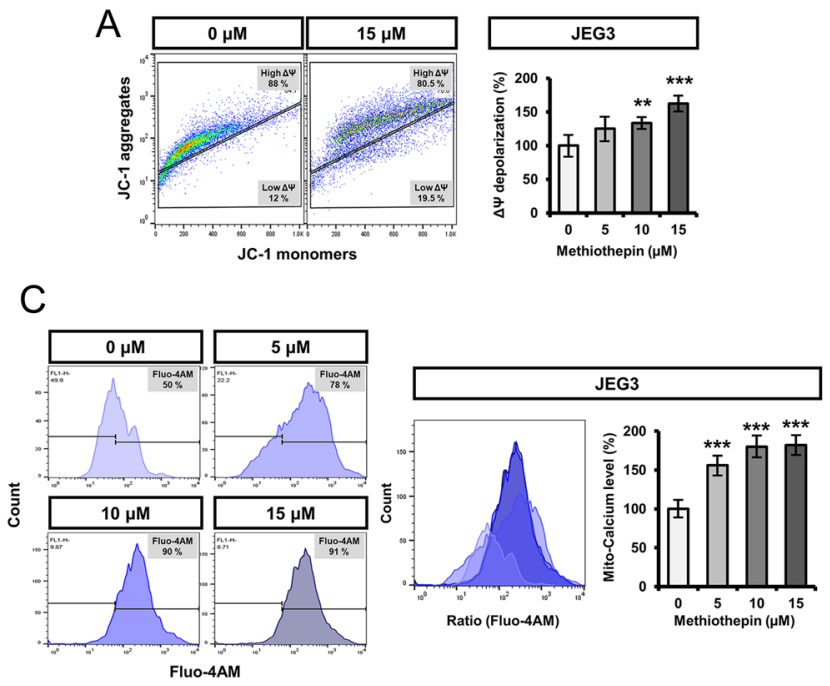

\section{Methiothepin compromises OXPHOS in mitochondria and glycolytic capacity in human placental choriocarcinoma cells}

Our previous data confirmed that methiothepin inhibited ATP production (Fig. 1) and induced mitochondrial stress (Fig. 3) against both placental choriocarcinoma cell lines. Therefore, we next evaluated the effect of methiothepin on the mitochondrial respiratory chain complexes as mitochondrial bioenergetics, considering the hypothesis that mitochondrial energy production is abrogated, thereby leading to metabolic shift and concomitant growth reduction in choriocarcinoma cells. We measured the activities of mitochondrial respiratory chain complex in response to each of the mitochondrial complex inhibitors including oligomycin, FCCP, rotenone, and antimycin A in methiothepin treated-choriocarcinoma cells. In both JEG3 and JAR cells, the activity of mitochondrial respiratory chain was attenuated by methiothepin, resulting in significant reduction of the OCR, revealing energy deprivation in placental choriocarcinoma cells (Fig. $4 \mathrm{~A}$ and $\mathrm{B})$. In particular, basal respiration, proton leak, maximal respiration, spare respiratory capacity, nonmitochondrial respiration, and ATP production were dramatically suppressed by methiothepin in JEG3 and JAR cells (Fig. 4C and D). In agreement with the OXPHOS results, energy phenotypes in JEG3 and JAR cells were shifted from energetic status to dormant, and mitochondrial respiration and extracellular acidification were reduced by methiothepin (Fig. 4E and F). Together, methiothepin attenuated the action of mitochondrial respiratory chain complexes, which triggered energy phenotypic alterations; the accompanying energy
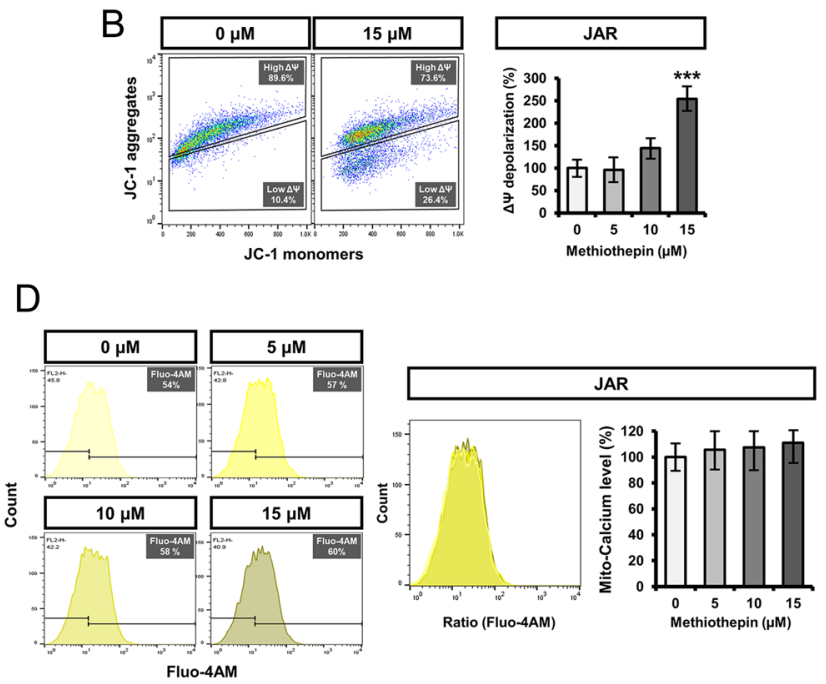

Figure 3 Effect of methiothepin on mitochondrial membrane rupture and calcium influx in human placental choriocarcinoma cells. (A and B) Depolarization of mitochondrial membrane potential analyzed by JC-1 staining in dose-dependent methiothepin-treated JEG3 (A) and JAR cells (B). (C and D) Mitochondrial calcium influx in methiothepin-treated JEG3 (C) and JAR (D) cells analyzed by Fluo-4 staining and flow cytometry. Results are presented as means \pm S.D. obtained from three independent experiments. The asterisk indicates statistically significant differences analyzed by Dunnett's test, one -way ANOVA $\left.{ }^{* *} P<0.01 ;{ }^{* * *} P<0.001\right)$. 

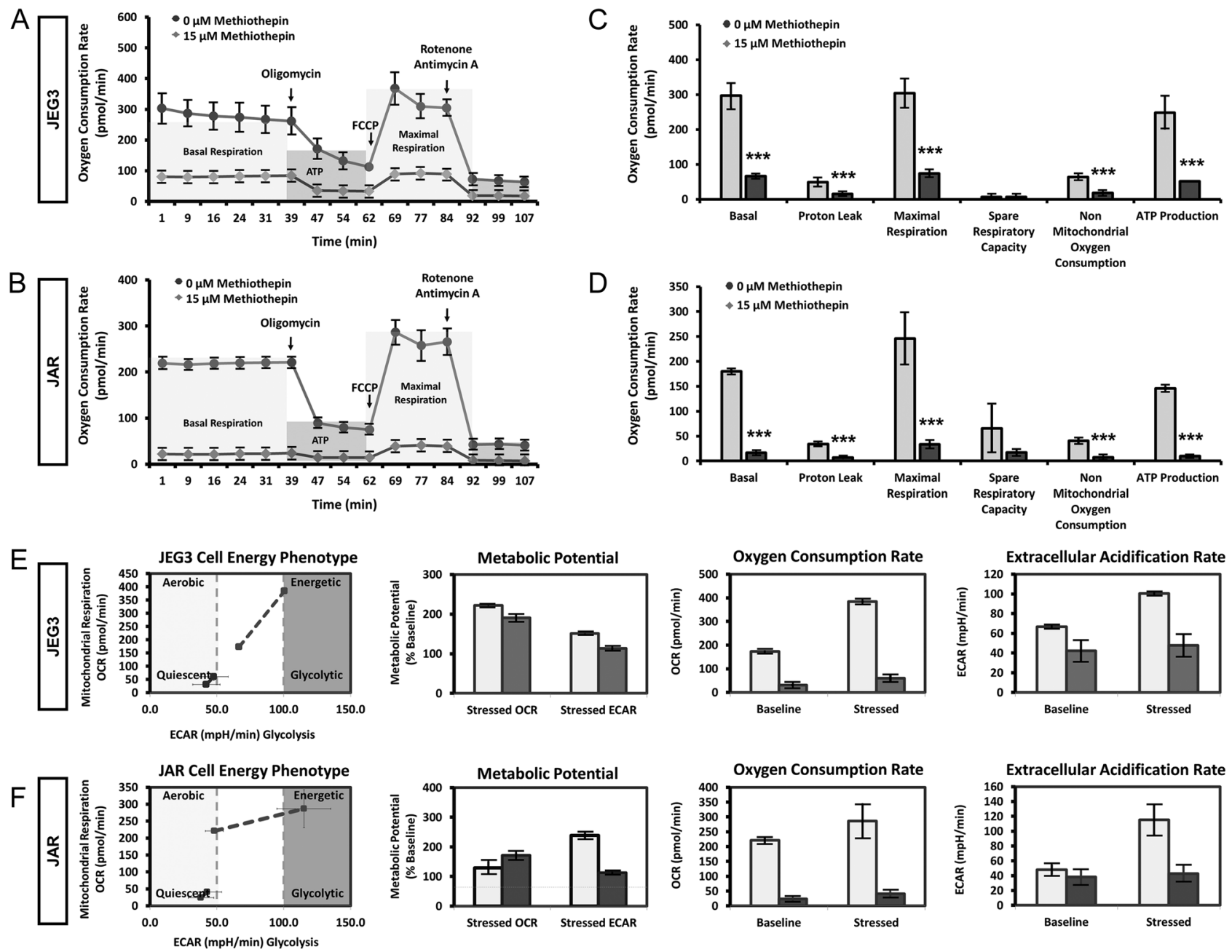

Figure 4 Effects of methiothepin on oxidative phosphorylation and metabolic alterations in human placental choriocarcinoma cells. (A and B) Oxidative phosphorylation in mitochondria analyzed by seahorse assay in vehicle- or methiothepin-treated JEG3 (A) and JAR (B) cells. (C and D) Various parameters for oxidative stress generated by seahorse assay in vehicle- or methiothepin-treated JEG3 (C) and JAR (D) cells such as basal level, proton leak, maximum respiration, spare respiratory capacity, non-mitochondrial oxygen consumption, and ATP production. (E and F) Energy phenotypes in JEG3 (E) and JAR (F) cells in response to the vehicle or methiothepin treatment. Results are presented as means \pm S.D. obtained from three independent experiments. The asterisk indicates statistically significant differences and was analyzed by Dunnett's test, one-way ANOVA $(* * * P<0.001)$.

deprivation resulted in reduced growth and activated apoptosis in the placental choriocarcinoma cells.

\section{Methiothepin triggers ER stress in human placental choriocarcinoma cells}

As the ER is functionally connected to the mitochondria, a property known as the mito-ER axis, we determined the effects of methiothepin on ER stress to demonstrate that systemic cellular stress results in anti-cancer effect in choriocarcinoma cells. According to the expression of ER stress regulatory proteins by methiothepin in JEG3 cells, GRP78, ATF4, p-PERK, and CHOP expression increased by 3.4-, 2.8-, 6.3-, and 5.7-fold, respectively, at the highest concentration of methiothepin compared to vehicle treatment (Fig. 5A). In JAR cells, GRP78,
ATF4, p-PERK, and CHOP expression were stimulated by $8.1-, 2.3-, 4.8-$, and 2.4 -fold compared to the control (Fig. 5B). Western blot and densitometry measurement confirmed that ER stress was induced by methiothepin in placental choriocarcinoma cells, which interacted with mitochondrial stress, eventually leading to proapoptotic effects.

\section{Methiothepin facilitates the paclitaxel response in human placental choriocarcinoma}

Considering that methiothepin is an FDA-approved drug, its pharmacological value as an anti-cancer drug is promising. Therefore, we evaluated the synergistic anti-cancer potential of methiothepin combined with paclitaxel in human placental choriocarcinoma 

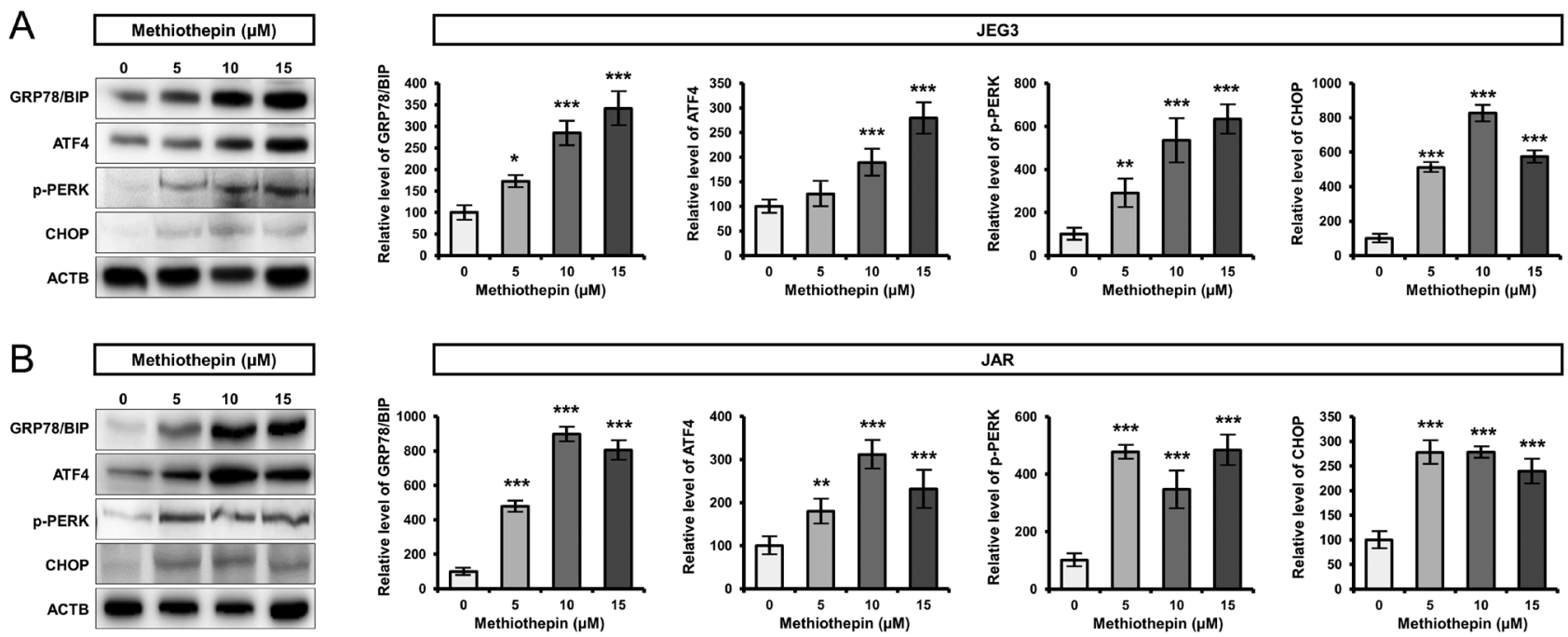

Figure 5 Effects of methiothepin on expression of ER stress regulatory proteins in human placental choriocarcinoma cells. (A and B) Immunoblot (left panel) of indicated proteins and densitometry analysis (right panel) of JEG3 (A) and JAR (B) cells by dose-dependent methionine treatment. GRP78/Bip, ATF4, p-PERK, and CHOP expression were evaluated as ES stress marker proteins. Results are presented as mean \pm s.D. obtained from three independent experiments. The asterisk indicates statistically significant differences analyzed by Dunnett's test, one-way ANOVA ( $P<$ $0.05 ; * * P<0.01 ; * * * P<0.001)$.

cells. According to cell viability, combination of $15 \mu \mathrm{M}$ methiothepin and 0.1 or $0.5 \mu \mathrm{M}$ paclitaxel synergistically reduced cell viability, compared to the case for treatment with either methiothepin or paclitaxel alone in JEG3 (Fig. 6A) and JAR cells (Fig. 6B). To support the viability data, we calculated the combination index using a computational software, CompuSyn. The combined treatments resulted in most of the dots showed a combination index below 1 , which implied that this combination exhibits synergy in JEG3 (Fig. 6A) and JAR cells (Fig. 6B). In agreement with the previous results, Hoechst and PI double staining demonstrated that drug combinations synergistically increased the proportions of late apoptotic and necrotic JEG3 cells (Fig. 6C). Moreover, in JAR cells, synergistic necrosis mainly occurred following treatment with the combination of methiothepin and paclitaxel, compared to the case for treatment with either methiothepin or paclitaxel alone (Fig. 6D). Therefore, methiothepin sensitized the paclitaxel response in both JEG3 and JAR cells, leading to synergistic anti-cancer activity.

\section{Discussion}

This is the first report to determine that methiothepin, a 5-HT receptor antagonist, has anti-cancer potential in human placental choriocarcinoma cells, and these effects are mostly induced through the mitochondrial respiratory complexes and its associated intracellular stresses, thereby leading to tumor suppression. Methiothepin is also known to inhibit human prostate cancer cells by stimulating oxidative stress, and to suppress ovarian cancer cells by mitochondria mediating metabolism
(Lee et al. 2020b, Yang et al. 2020). Considering that methiothepin is an FDA-approved psychotropic agent as well as a 5-HT receptor antagonist, our study provides new insights into the repositioning of this drug as an anti-cancer agent, in particular, for treating placental choriocarcinoma.

$5-\mathrm{HT}$ consists of seven subgroups $\left(5-\mathrm{HT} 1-5-\mathrm{HT}_{7}\right)$, which mostly function as neurotransmitters distributed in the CNS. However, recent articles have described its diverse functions beyond its role in the CNS, for instance, in hematopoietic differentiation, vascular maintenance, inflammatory response, and tissue regeneration (Lv \& Liu 2017). Among them, 5- $\mathrm{HT}_{2 \mathrm{~A}}$, found to promote cell growth, is seen in various types of cells including skeletal and vascular muscle cells (Watts 1996, GuilletDeniau et al. 1997). Moreover, the mitogenic activity of $5-\mathrm{HT}$ through $5-\mathrm{HT}_{2 \mathrm{~A}}$ receptor has been explained as being mediated via activation of JAK-STAT and ERKMAPK signal transduction (Guillet-Deniau et al. 1997, Grewal et al. 1999, Watts et al. 2001).

Many articles have elucidated 5-HT as a physiological regulator in the reproductive system. During the progression of pregnancy, it plays an important role in the regulation of de novo synthesis of estrogen, and loss of control in serotonergic system potentially causes disturbance of reproductive hormone which eventually leads to functional failure in placenta (Hudon Thibeault etal. 2019). 5-HT and its receptors have been identified as growth promoters with mitogenic effects in reproductive cells such as rat jejunal crypt cells (Tutton \& Barkla 1987), bovine placental cells (Fecteau \& Eiler 2001), and fetal tissues (Lauder et al. 1981). Other than its role in normal conditions, 5-HT also shows tumor promotion 


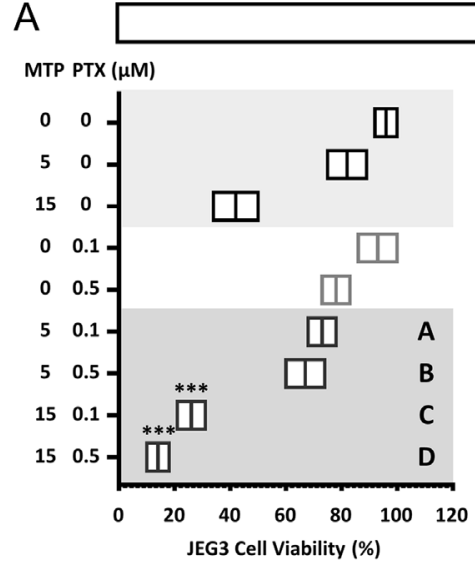

JEG3

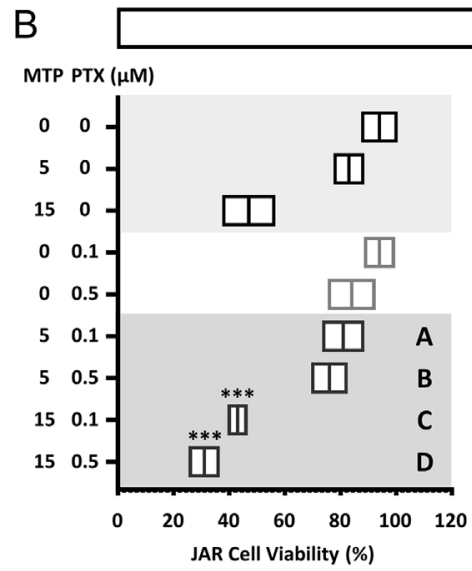

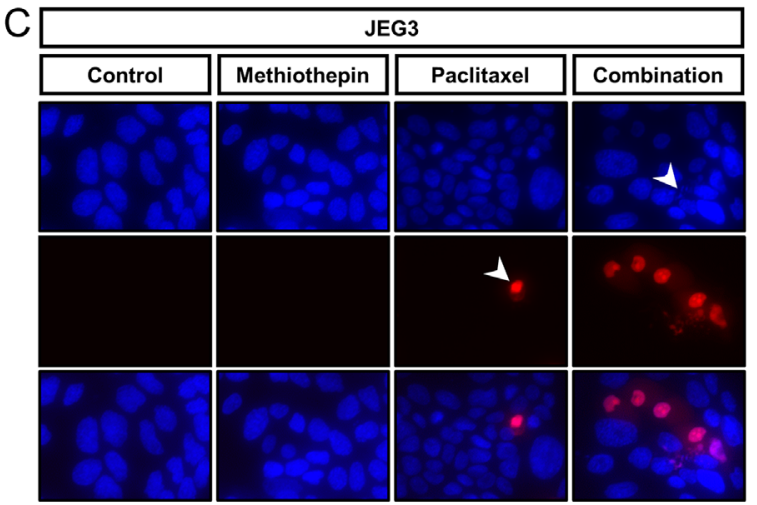

Fraction Affected (FA)
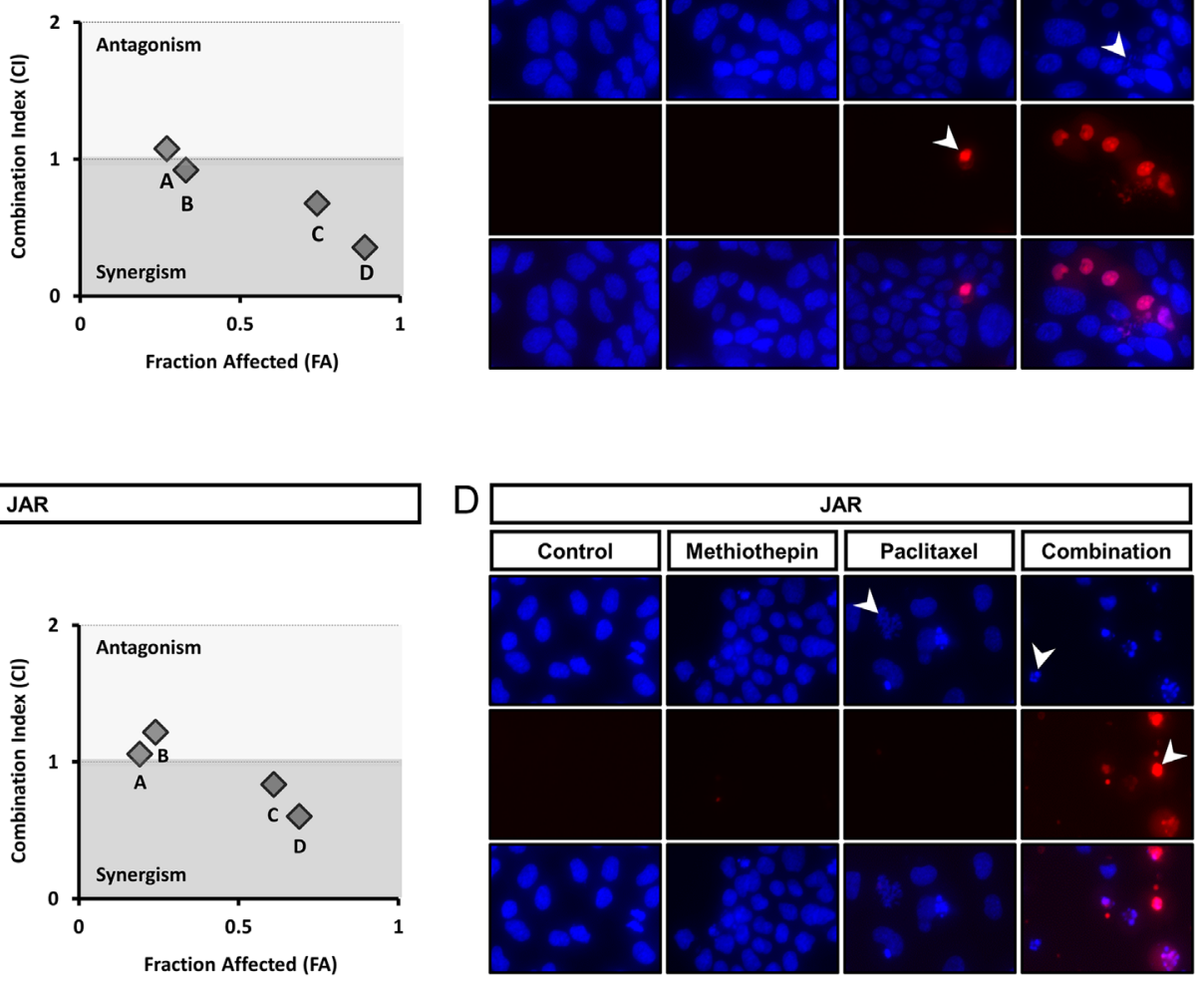

Figure 6 Synergistic anti-cancer effect of methiothepin and paclitaxel in human placental choriocarcinoma cells. (A and B) Cell viability (left panel) and combination index (right panel) in JEG3 (A) and JAR (B) cells in response to the indicated drug treatment. Combination index calculated by CompuSyn software in response to treatment with either methiothepin (MTP) or paclitaxel (PTX) alone or with a combination of methiothepin and paclitaxel in the cells. (C and D) Hoechst and PI analysis performed to analyze response to treatment with either methiothepin or paclitaxel alone or with a combination of methiothepin and paclitaxel in JEG3 (C) and JAR (D) cells. Results are presented as means \pm S.D. obtained from three independent experiments. The asterisk indicates statistically significant differences analyzed by Dunnett's test, one-, or two-way ANOVA (***P $<0.001)$.

activity in different types of carcinogenesis, such as in adenocarcinoma (Tutton \& Barkla 1987), pancreatic carcinoid cells (Ishizuka et al. 1992), and small cell lung cancer (Cattaneo et al. 1993). In particular, proliferation of placental choriocarcinoma cells was also affected by 5-HT and its receptors through mitogenic function, and the $5-\mathrm{HT}$ receptor has been identified to be localized in the plasma membrane of placental choriocarcinoma cells (Sonier et al. 2005). The implication of the interaction between 5-HT and placental choriocarcinoma is also supported by its role in the regulation of the human chorionic gonadotrophin (hCG) hormone, which is a diagnostic biomarker for therapeutic outcome and tumor stage in placental choriocarcinoma, as well as in pathological pregnancy (Betz \& Fane 2020, Lee et al. 2020a). 5-HT stimulates hCG secretion in Leydig cells (Tinajero et al. 1992), and the positive expression of G-protein coupled receptors (GPCRs) including hCG receptors has been reported in reproductive malignant tumors of the ovary, endometrium, and breast (Lenhard et al. 2012, Kolbl et al. 2018, Betz \& Fane 2020). This interaction between serotonergic system and placental hormone constitute the basis for our hypothesis that 5-HT receptor antagonist affects placental functions, halting the progression of placental choriocarcinoma.

Our novel findings suggest that methiothepin, a 5-HT receptor antagonist suppresses placental choriocarcinoma cells not only through mitochondrial biogenesis but also through mitochondrial related intracellular stresses via caspase activation and apoptosis pathways. In particular, the robust reduction of overall mitochondrial functions and the metabolic shift by 5-HT receptor antagonist provides new insight into a potential therapeutic strategy against placental choriocarcinoma along with the findings of previous articles. In fact, if the physiological functions are controlled by 5-HT receptor, its mechanisms are involved in the mitochondrial energetic functions and oxidative process. For instance, 
in renal proximal tubular cells, $5-\mathrm{HT}_{2 \mathrm{~A}}$ receptor stimulates mitochondrial biogenesis via peroxisome proliferator-activated receptor-gamma coactivator (PGC)- $1 \alpha$ and improves the ability to recover from kidney injury (Harmon et al. 2016, Gibbs et al. 2018). 5-HT also contributes to increasing the cell viability through $5-\mathrm{HT}_{2 \mathrm{~B}}$ receptors and internal PI3K-MAPK pathways in cardiomyocytes (Nebigil et al. 2003). In the cortical neurons, stimulation of 5- $\mathrm{HT}$ and $5-\mathrm{HT}_{2 \mathrm{~A}}$ leads to basal OCR upregulation, which implies the high energy demand that in turn induces the electron transport chain activity, leading to mitochondrial functions. In addition, $5-\mathrm{HT}$ and $5-\mathrm{HT}_{2 \mathrm{~A}}$ agonists enhance ATP generation, OXPHOS levels, maximal respiration, and oxidative capacity (Fanibunda et al. 2019). In addition, 5-HT directly activates mitochondrial respiration and glycolysis in breast cancer cells through STAT3 and ERK pathway (Sola-Penna et al. 2020), and also induces superoxide anion production in pulmonary arteries (Genet et al. 2017). Oxidative respiration and physiological functions mediated by serotonin through mitochondria regardless of normal and pathological mechanisms have been elucidated, which means that these mechanisms may actively be targeted for therapeutic purposes.

In our data, we found that even after treatment with $15 \mu \mathrm{M}$ of methiothepin in HPC cells, metabolic indicators, including oxidative phosphorylation, are still damaged in living cells. That is, a significant decrease in cell viability identified by longer treatment with methiothepin suggests that it is associated with mitochondrial dysfunction by methiothepin. It was first identified in this study that methiothepin can affect mitochondrial OXPHOS, but it is known in some types of cells that serotonin can promote mitochondrial OXPHOS through receptor mediation. Therefore, it is speculated that methiothepin, as an antagonist of the serotonin receptor, inhibits its activity and affects mitochondrial OXPHOS. In detail, serotonin increases mitochondrial biogenesis, oxidative capacity, and ATP levels by mediating PLC and MAPK signaling in neuronal cells via serotonin receptors belonging to the GPCRs (Fanibunda et al. 2019). Serotonin, particularly in breast cancer cells, promotes mitochondrial biogenesis through the adenylyl cyclase/PKA pathway, associated with cancer progression (Sola-Penna et al. 2020). In addition, PPAR $\gamma$-mediated pathways, which are regulated by the activity of serotonin receptors, may also be associated with mitochondrial OXPHOS regulation (Harmon et al. 2016). This study did not confirm how methiothepin regulates mitochondrial OXPHOS. As demonstrated in several carcinomas, inhibition of the signaling pathway that induces mitochondrial biogenesis through suppressing GPCRs seems to be a potent mechanism for methiothepin, but the specific mechanism of

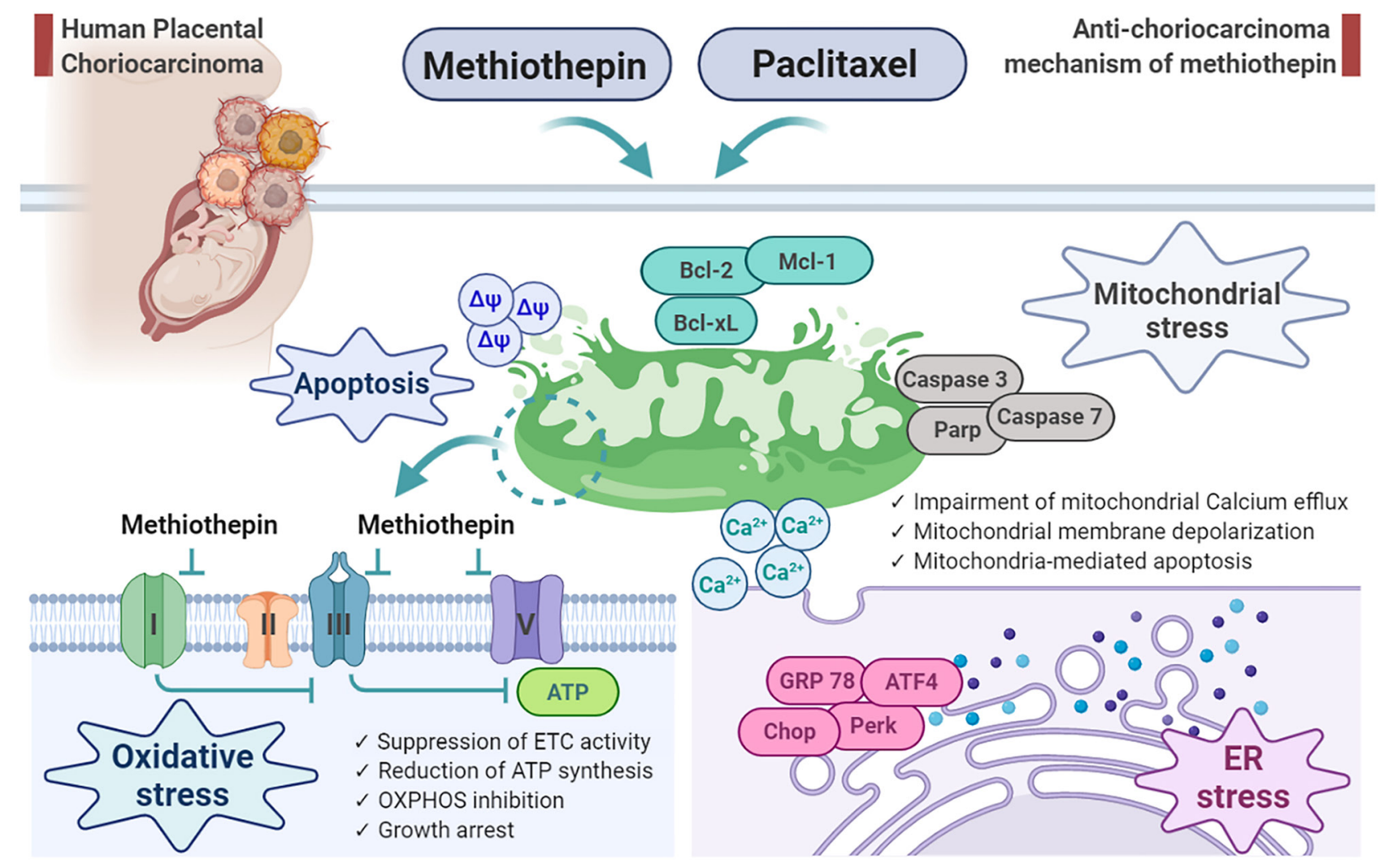

Figure 7 Schematic diagram of the anti-cancer mechanisms by which methiothepin targets human placental choriocarcinoma cells. 
methiothepin for mitochondrial respiration in HPC requires further study.

In agreement with the crucial role of $5-\mathrm{HT}$ in mitochondria, upregulation of 5-HT signaling influences the Warburg effect under metabolic stress, contributing to pancreatic tumor growth (Jiang et al. 2017). In addition, the $5-\mathrm{HT}_{3}$ receptor selective antagonist Y25130 was described as an anti-tumorigenic factor and apoptosis inducer, whereas the $5-\mathrm{HT}_{3}$ receptor selective agonist phenylbiguanide displays mitogenic function and promotes the growth of colon cancer cells along with (Ataee et al. 2010). Our study found that methiothepin induced metabolic modification and apoptosis in placental choriocarcinoma cells, which most likely resulted from mitochondrial and ER stress. Through the implication between mitochondrial stress and tremendous subsequent cellular effects on calcium ion levels, mitochondrial membrane potential, and electron transport chain activities, leading to interruption of the activity of mitochondrial respiratory complexes by methiothepin, we highlighted the robust anti-tumorigenic activity of methiothepin in human placental choriocarcinoma cells.

The mitochondrial membrane potential (MMP) is transmembrane potential energy for the gradient of the hydrogen ion $(\Delta \mu \mathrm{H}+)$ localized on the inner mitochondrial membrane (Zorova et al. 2018). The MMP control mechanism includes both proton pumps and discharge of their potential, and if minor discharges can reduce harmful ROS production to some extent, optimal MMP acts as a 'mild uncouplers', defense mechanisms (Korshunov et al. 1997), which, in turn, can be achieved the ROS reduction simultaneously with ATP production to maintain cellular homeostasis (Cunha et al. 2011). Proton pump generation by MMP, in particular, is a fundamental component for the energy storage process during oxidative phosphorylation (Perry et al. 2011, Zorova et al. 2018). Thus, once the mitochondrial membrane potential is disrupted, retardation in mitochondrial transport of positive charge elements occurs, which disturb an essential transport that generates energy source throughout the mitochondrial complexes (Zorova et al. 2018), consequently contributing to inhibit oxidative phosphorylation processes. Besides, MMP is a common topic in cancer research, and due to its physiological characteristics that cancer cells (approximately $220 \mathrm{mV}$ ) have higher hyperpolarized membrane potential than normal cells (approximately $140 \mathrm{mV}$ ), only cancer cells can be expected to be targeted by the drug. In our data, methiothepin inhibits mitochondrial membrane potential and calcium levels, thereby suppressing the OCR levels suggest that mitochondria in placental choriocarcinoma is involved in the drug mechanism by methiothepin.

Overall, our results regarding the novel pharmacological effect of methiothepin in placental choriocarcinoma indicate the active interaction between mitochondrial stress and tremendous subsequent cellular effects, such as those on the calcium ion levels, membrane depolarization, and electron transport chain activities as described in Fig. 7. Thus, we suggest that the mitochondrial dysfunction is involved in the methiothepin-mediated anti-cancer function, thereby leading to the suppression of the activity of the mitochondrial respiratory system and concomitant reduction of tumorigenesis in placental choriocarcinoma cells. Moreover, through our findings on the increased sensitization of placental choriocarcinoma cells to paclitaxel by methiothepin, we highlight robust economic and academic advantages associated with the repositioning of methiothepin as an anti-cancer agent; thus, different functional drug combinations involving FDA-approved agents may serve as potential agents for treating placental choriocarcinoma, and possibly, other cancers.

\section{Declaration of interest}

The authors declare that there is no conflict of interest that could be perceived as prejudicing the impartiality of the research reported.

\section{Funding}

This research was supported by a grant of the National Research Foundation of Korea (NRF) grant, funded by the Ministry of Science and ICT(MSIT) (grant number: 2018R1C1B6009048).

\section{Author contribution statement}

G S, W L and J Y L conceived and designed the culture experiments, the cell culture methodology, and other all experiments. J Y L collected experimental samples and conducted all experiments. J Y L, W L, and G S analyzed, interpreted the data and contributed to the development of the manuscript. All authors contributed to its critical review and agreed on the final version.

\section{References}

Al-Khan A, Bulmer JN, Chantraine F, Chen CP, Chen Q, Collins S, Cotechini T, Fitzgerald JS, He M, Holland O et al. 2013 IFPA Meeting 2012 Workshop Report III: trophoblast deportation, gestational trophoblastic disease, placental insufficiency and fetal growth restriction, trophoblast over-invasion and accreta-related pathologies, placental thrombosis and fibrinolysis. Placenta 34 (Supplement) S11-S16. (https:// doi.org/10.1016/j.placenta.2012.11.018)

Ataee R, Ajdary S, Zarrindast M, Rezayat M, Shokrgozar MA \& Ataee A 2010 Y25130 hydrochloride, a selective 5HT3 receptor antagonist has potent antimitogenic and apoptotic effect on HT29 colorectal cancer cell line. European Journal of Cancer Prevention 19 138-143. (https:// doi.org/10.1097/CEJ.0b013e3283354901)

Betz D \& Fane K 2020 Human Chorionic Gonadotropin (HCG). Treasure Island (FL): StatPearls.

Bowen JM, Chamley L, Mitchell MD \& Keelan JA 2002 Cytokines of the placenta and extra-placental membranes: biosynthesis, secretion and roles in establishment of pregnancy in women. Placenta 23 239-256. (https://doi.org/10.1053/plac.2001.0781) 
Carson DD, Bagchi I, Dey SK, Enders AC, Fazleabas AT, Lessey BA \& Yoshinaga K 2000 Embryo implantation. Developmental Biology 223 217-237. (https://doi.org/10.1006/dbio.2000.9767)

Cattaneo MG, Codignola A, Vicentini LM, Clementi F \& Sher E 1993 Nicotine stimulates a serotonergic autocrine loop in human small-cell lung carcinoma. Cancer Research 53 5566-5568.

Cunha FM, Caldeira da Silva CC, Cerqueira FM \& Kowaltowski AJ 2011 Mild mitochondrial uncoupling as a therapeutic strategy. Current Drug Targets 12 783-789. (https://doi.org/10.2174/138945011795528778)

Fanibunda SE, Deb S, Maniyadath B, Tiwari P, Ghai U, Gupta S, Figueiredo D, Weisstaub N, Gingrich JA, Vaidya ADB et al. 2019 Serotonin regulates mitochondrial biogenesis and function in rodent cortical neurons via the 5-HT2A receptor and SIRT1-PGC-1alpha axis. PNAS 116 11028-11037. (https://doi.org/10.1073/pnas.1821332116)

Fecteau KA \& Eiler H 2001 Placenta detachment: unexpected high concentrations of 5-hydroxytryptamine (serotonin) in fetal blood and its mitogenic effect on placental cells in bovine. Placenta 22 103-110. (https://doi.org/10.1053/plac.2000.0596)

Genet N, Billaud M, Rossignol R, Dubois M, Gillibert-Duplantier J, Isakson BE, Marthan R, Savineau JP \& Guibert C 2017 Signaling pathways linked to serotonin-induced superoxide anion production: a physiological role for mitochondria in pulmonary arteries. Frontiers in Physiology 8 76. (https://doi.org/10.3389/fphys.2017.00076)

Gibbs WS, Collier JB, Morris M, Beeson CC, Megyesi J \& Schnellmann RG 2018 5-HT1F receptor regulates mitochondrial homeostasis and its loss potentiates acute kidney injury and impairs renal recovery. American Journal of Physiology: Renal Physiology 315 F1119-F1128. (https://doi. org/10.1152/ajprenal.00077.2018)

Grewal JS, Mukhin YV, Garnovskaya MN, Raymond JR \& Greene EL 1999 Serotonin 5-HT2A receptor induces TGF-beta1 expression in mesangial cells via ERK: proliferative and fibrotic signals. American Journal of Physiology 276 F922-F930. (https://doi.org/10.1152/ ajprenal.1999.276.6.F922)

Guillet-Deniau I, Burnol AF \& Girard J 1997 Identification and localization of a skeletal muscle secrotonin 5-HT2A receptor coupled to the Jak/STAT pathway. Journal of Biological Chemistry 272 14825-14829. (https://doi. org/10.1074/jbc.272.23.14825)

Harmon JL, Wills LP, McOmish CE, Demireva EY, Gingrich JA, Beeson CC \& Schnellmann RG 2016 5-HT2 receptor regulation of mitochondrial genes: unexpected pharmacological effects of agonists and antagonists. Journal of Pharmacology and Experimental Therapeutics 357 1-9. (https://doi.org/10.1124/jpet.115.228395)

Hudon Thibeault AA, Sanderson JT \& Vaillancourt C 2019 Serotoninestrogen interactions: what can we learn from pregnancy? Biochimie $\mathbf{1 6 1}$ 88-108. (https://doi.org/10.1016/j.biochi.2019.03.023)

Ishizuka J, Beauchamp RD, Townsend CM, Jr, Greeley GH, Jr \& Thompson JC 1992 Receptor-mediated autocrine growth-stimulatory effect of 5-hydroxytryptamine on cultured human pancreatic carcinoid cells. Journal of Cellular Physiology 150 1-7. (https://doi.org/10.1002/ jcp.1041500102)

Jiang SH, Li J, Dong FY, Yang JY, Liu DJ, Yang XM, Wang YH, Yang MW, Fu XL, Zhang XX et al. 2017 Increased serotonin signaling contributes to the Warburg effect in pancreatic tumor cells under metabolic stress and promotes growth of pancreatic tumors in mice. Gastroenterology 153 277.e19-291.e19. (https://doi.org/10.1053/j.gastro.2017.03.008)

Kolbl AC, Schlenk K, Behrendt N \& Andergassen U 2018 The importance of hCG in human endometrial adenocarcinoma and breast cancer. International Journal of Biological Markers 33 33-39. (https://doi. org/10.5301/ijbm.5000290)

Korshunov SS, Skulachev VP \& Starkov AA 1997 High protonic potential actuates a mechanism of production of reactive oxygen species in mitochondria. FEBS Letters 416 15-18. (https://doi.org/10.1016/s00145793(97)01159-9)

Lauder JM, Wallace JA \& Krebs H 1981 Roles for serotonin in neuroembryogenesis. Advances in Experimental Medicine and Biology 133 477-506. (https://doi.org/10.1007/978-1-4684-3860-4_28)

Lee JY, Park H, Lim W \& Song G 2020a Alpha,beta-thujone suppresses human placental choriocarcinoma cells via metabolic disruption. Reproduction 159 745-756. (https://doi.org/10.1530/REP-20-0018)

Lee JY, Yang C, Lim W \& Song G $2020 b$ Methiothepin suppresses human ovarian cancer cell growth by repressing mitochondrion-mediated metabolism and inhibiting angiogenesis in vivo. Pharmaceutics 12686. (https://doi.org/10.3390/pharmaceutics12070686)
Lenhard M, Tsvilina A, Schumacher L, Kupka M, Ditsch N, Mayr D, Friese K \& Jeschke U 2012 Human chorionic gonadotropin and its relation to grade, stage and patient survival in ovarian cancer. BMC Cancer 122. (https://doi.org/10.1186/1471-2407-12-2)

Lv J \& Liu F 2017 The role of serotonin beyond the central nervous system during embryogenesis. Frontiers in Cellular Neuroscience 11 74. (https:// doi.org/10.3389/fncel.2017.00074)

Matsuyama S \& Reed JC 2000 Mitochondria-dependent apoptosis and cellular pH regulation. Cell Death and Differentiation 7 1155-1165. (https://doi.org/10.1038/sj.cdd.4400779)

Nebigil CG, Etienne N, Messaddeq N \& Maroteaux L 2003 Serotonin is a novel survival factor of cardiomyocytes: mitochondria as a target of 5-HT2B receptor signaling. FASEB Journal 17 1373-1375. (https://doi. org/10.1096/fj.02-1122fje)

Perry SW, Norman JP, Barbieri J, Brown EB \& Gelbard HA 2011 Mitochondrial membrane potential probes and the proton gradient: a practical usage guide. BioTechniques 5098-115. (https://doi.org/10.2144/ 000113610)

Pithadia AB \& Jain SM 2009 5-Hydroxytryptamine receptor subtypes and their modulators with therapeutic potentials. Journal of Clinical Medicine Research 1 72-80. (https://doi.org/10.4021/jocmr2009.05.1237)

Rosario FJ, Gupta MB, Myatt L, Powell TL, Glenn JP, Cox L \& Jansson T 2019 Mechanistic target of rapamycin complex 1 promotes the expression of genes encoding electron transport chain proteins and stimulates oxidative phosphorylation in primary human trophoblast cells by regulating mitochondrial biogenesis. Scientific Reports 9 246. (https:// doi.org/10.1038/s41598-018-36265-8)

Sola-Penna M, Paixao LP, Branco JR, Ochioni AC, Albanese JM, Mundim DM, Baptista-de-Souza D, Figueiredo CP, Coelho WS, Marcondes MC et al. 2020 Serotonin activates glycolysis and mitochondria biogenesis in human breast cancer cells through activation of the Jak1/STAT3/ERK1/2 and adenylate cyclase/PKA, respectively. British Journal of Cancer 122 194-208. (https://doi.org/10.1038/s41416-019-0640-1)

Sonier B, Lavigne C, Arseneault M, Ouellette R \& Vaillancourt C 2005 Expression of the 5-HT2A serotoninergic receptor in human placenta and choriocarcinoma cells: mitogenic implications of serotonin. Placenta $\mathbf{2 6}$ 484-490. (https://doi.org/10.1016/j.placenta.2004.08.003)

Tinajero JC, Fabbri A \& Dufau ML 1992 Regulation of corticotropinreleasing factor secretion from Leydig cells by serotonin. Endocrinology 130 1780-1788. (https://doi.org/10.1210/endo.130.4.1312425)

Tutton PJ \& Barkla DH 1987 Biogenic amines as regulators of the proliferative activity of normal and neoplastic intestinal epithelial cells (review). Anticancer Research 7 1-12.

Watts SW 1996 Serotonin activates the mitogen-activated protein kinase pathway in vascular smooth muscle: use of the mitogen-activated protein kinase kinase inhibitor PD098059. Journal of Pharmacology and Experimental Therapeutics 279 1541-1550.

Watts SW, Yang P, Banes AK \& Baez M 2001 Activation of Erk mitogenactivated protein kinase proteins by vascular serotonin receptors. Journal of Cardiovascular Pharmacology 38 539-551. (https://doi. org/10.1097/00005344-200110000-00006)

Yang C, Song G \& Lim W 2020 Methiothepin mesylate causes apoptosis of human prostate cancer cells by mediating oxidative stress and mitochondrial dysfunction. Free Radical Biology and Medicine $\mathbf{1 5 0}$ 12-22. (https://doi.org/10.1016/j.freeradbiomed.2020.01.187)

Yung HW, Colleoni F, Dommett E, Cindrova-Davies T, Kingdom J, Murray AJ \& Burton GJ 2019 Noncanonical mitochondrial unfolded protein response impairs placental oxidative phosphorylation in earlyonset preeclampsia. PNAS 116 18109-18118. (https://doi.org/10.1073/ pnas.1907548116)

Zorova LD, Popkov VA, Plotnikov EY, Silachev DN, Pevzner IB, Jankauskas SS, Babenko VA, Zorov SD, Balakireva AV, Juhaszova M et al. 2018 Mitochondrial membrane potential. Analytical Biochemistry 552 50-59. (https://doi.org/10.1016/j.ab.2017.07.009)

Received 6 July 2020

First decision 6 August 2020

Revised Manuscript received 4 September 2020

Accepted 23 September 2020 